\title{
Coexistence of systemic lupus erythematosus, Hashimoto's thyroiditis, and bilateral breast cancer in the same patient: a random association?
}

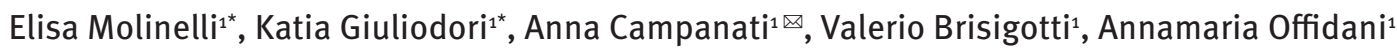

\begin{abstract}
Estrogens influence many physiological processes and play a crucial role in the development of several diseases, including autoimmune disorders and hormone-sensitive cancers. Systemic lupus erythematosus is one of the most common systemic autoimmune rheumatic diseases affecting young and middle-aged females. The coexistence of multiple autoimmune disorders is well recognized, whereas the association between systemic lupus erythematosus and malignancies, especially hormone-sensitive cancers, remains enigmatic. We report the unusual case of a middle-aged woman that presented with concomitance of lupus erythematosus, Hashimoto's thyroiditis, and bilateral breast cancer.
\end{abstract}

Keywords: estrogens, breast cancer, lupus erythematosus, Hashimoto’s thyroiditis

Received: 16 April 2015 | Returned for modification: 12 May 2015 | Accepted: 3 June 2015

\section{Introduction}

Systemic lupus erythematosus (SLE) is a common multiorgan autoimmune disease with a prevalence of about 20 to 150 per 100,000 that predominantly affects women of reproductive age. The etiology remains largely unknown. Environmental triggers, susceptibility genes, immunological abnormalities, and hormonal factors are involved in SLE pathogenesis. Estrogens strikingly influence susceptibility to SLE, as evidenced by female predominance and unusual presentation during the prepubertal and postmenopausal period (1).

SLE has frequently been reported in association with a variety of organ-specific autoimmune disorders, especially vitiligo, inflammatory bowel disease, and autoimmune thyroiditis (2-4).

The relation between cancer and immunologically mediated inflammatory diseases has been intensively discussed. Several autoimmune conditions, including SLE, have consistently been associated with increased risk of hematological (lymphoid) malignancies, particularly non-Hodgkin lymphoma (NHL). Conversely, the incidence of solid tumors, mostly breast cancer, among patients with SLE is a controversial issue (5).

We present the unusual case of a 51-year-old woman with coexisting systemic lupus erythematosus, Hashimoto's thyroiditis, and breast cancer, analyzing the possible associations between these diseases.

\section{Case report}

The 51-year-old patient was diagnosed with chronic cutaneous lupus erythematosus (CCLE) in 2001 at age 38. Well-defined erythematous, infiltrated, scaly patches were confined to sun-exposed skin, particularly the face, neck, upper trunk, and back (Figs. 1 and 2). Routine laboratory findings including complete blood cell count and liver and renal function tests were normal. A serological test revealed 1:320 antinuclear antibodies (ANA) titers. The diagnosis of disseminated discoid lupus erythematosus (DLE) was confirmed by histological and direct immunofluorescence exami- nation. Topical high-potency corticosteroids were used without satisfactory improvement.

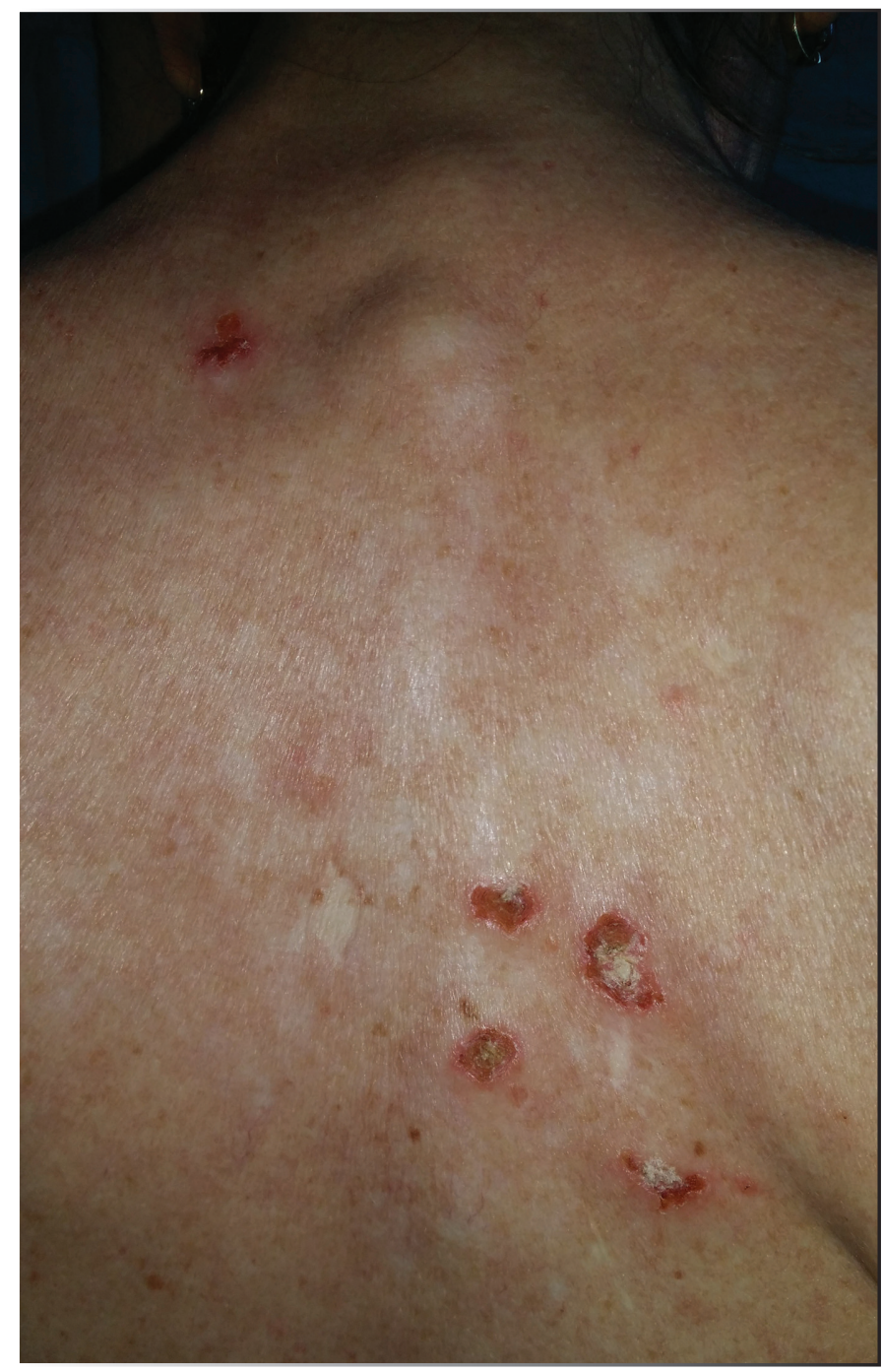

Figure 1 | Erythematous, infiltrated, scaly patches on the upper back. 


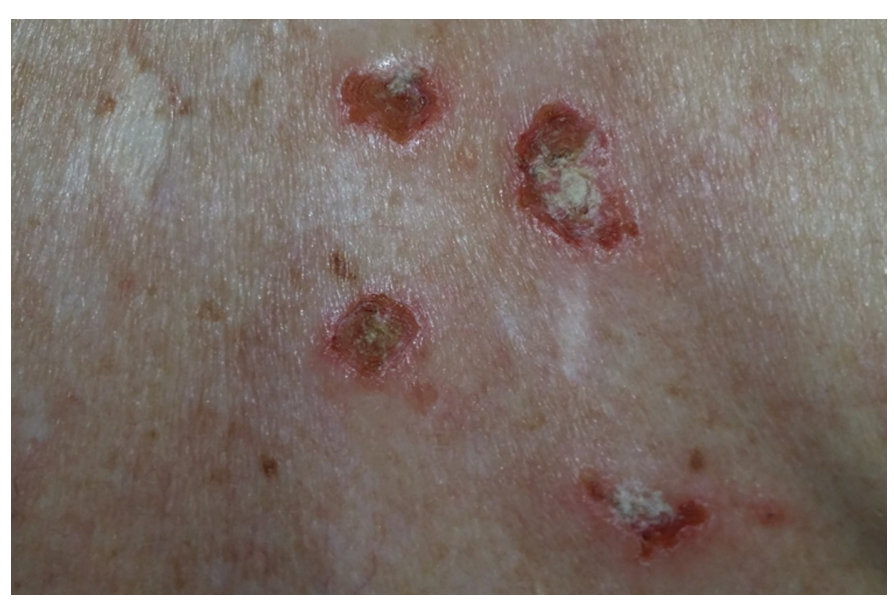

Figure 2 | Magnification of a cutaneous lesion.

Despite the absence of personal and familial risk factors, 5 months later invasive ductal breast cancer was detected on the right side. The patient underwent surgical treatment with postoperative radiotherapy and also received adjuvant hormonal therapy. During this period the DLE was slightly exacerbated. Systemic corticosteroids were administered with limited success because recurrence of cutaneous lesions was observed after a few months.

In 2010, invasive ductal breast cancer was diagnosed on the left side. Breast conservative surgery and chemotherapy were performed. During the chemotherapeutic regimen, CCLE skin lesions improved dramatically.

After 20 months of stable disease, CCLE relapsed and progressed. Clinical examination revealed multiple, annular, erythematous plaques on the face, upper part of the back, and arms. She also developed disfiguring scarring alopecia.

Laboratory investigations showed mild leukopenia and anemia, and slightly elevated erythrocyte sedimentation rate $(56 \mathrm{~mm} / \mathrm{hr})$. A serological test revealed antinuclear antibody (ANA), 1:640, with a homogeneous pattern. Anti-DNA antibody, anti-Smith, SS-A and SS-B antibody, anti-RNP IgG, and cardiolipin IgG and IgA antibody were not detected. Anti-phospholipid antibodies (lupus anticoagulant antibodies and anti- $\beta$-2 glycoprotein I) were identified. Complement components ( $\mathrm{C}_{3}$ and $\left.\mathrm{C}_{4}\right)$ were slightly decreased. In addition, pain and functional disabilities affecting the joints of the hands and feet were also observed.

Histological cutaneous examination revealed epidermal atrophy, and superficial and deep perivascular and perifollicular dermatitis with focally increased dermal mucin highlighted by Alcian blue stain, suggesting lupus erythematosus (LE). Direct immunofluorescence of lesional skin showed immunoglobulins (IgG, IgA) and $\mathrm{C}_{3}$ in a granular band-like pattern at the dermo-epidermal junction. A diagnosis of SLE with cutaneous, hematological, and joint involvement was made by a rheumatologist.

Oral prednisone in combination with antimalarial therapy was initially started and gradually tapered over the next 4 months. Within 12 weeks, the cutaneous lesions and hematological values improved significantly. In December 2013 the patient was tested for thyroid antibodies and showed high levels of serum anti-thyroperoxidase and anti-thyroglobulin autoantibodies. Thyroid hormones (fT3, fT4) were normal with TSH levels slightly increased. A diagnosis of autoimmune thyroiditis (AT)-specifically, Hashimoto's thyroiditis with subclinical hypothyroidism-was established.

\section{Discussion}

The overall cancer risk in SLE is increased compared to general population. The loss of self-tolerance and the exposure to cytotoxic drugs seems to play a central role in the development of sitespecific cancer (5). SLE patients appear to have a considerable increased risk of hematological malignancies, particularly nonHodgkin lymphoma, leukemia, and cancers of the vulva, lung, thyroid, and possibly liver. Conversely, most authors agree that SLE patients present a decreased risk of hormone-sensitive cancers, including ovarian cancer, endometrial cancer, and breast cancer. Breast cancer is one of the major causes of cancer-related morbidity and mortality among women worldwide (6).

The risk of breast cancer is traditionally associated with hormonal factors, especially cumulative estrogen exposure (due to reproductive history and hormonal therapy) (7).

The breast cancer risk in SLE patient appears decreased both in pre-menopausal and post-menopausal women (8).

The paradoxical reduction of the reproductive malignancy incidence in females with SLE is coherent with the suggestion that women suffering from SLE have been shown to have earlier menopause compared to the general female population. However alterations in estrogen metabolism and/or other hormones, and other additional factors, including genetic susceptibility and medication exposure, seem to be related to breast carcinogenesis in the SLE population. Drug factors contributing to a protective effect for certain cancers could be present in SLE: treatment with anti-malarial drugs has been proposed as a cause of the decreased risk of breast cancer in SLE patients. It has been hypothesized that antimalarials have a potential role in cancer treatment through a cell-death process called autophagy. In addition, aspirin and nonsteroidal anti-inflammatory drugs may be beneficial in reducing breast cancer risk, as in other tumors (9).

Thus, carcinogenesis of breast cancer in SLE women in not thoroughly understood and remains relatively unclear because it does not seem completely related to traditional risk factors. Conversely, a subgroup with a higher risk of breast tumors among patients with SLE has already been reported (6).

Our patient that presented with bilateral breast cancer could belong to this subset. It may be hypothesized that SLE women with higher breast cancer risk show rare genetic polymorphisms involving estrogen receptors, or metabolic pathway, which could lead patients to higher endogenous estrogen exposure throughout their reproductive lifetime.

Alternatively, genetic influences predisposing to both autoimmune diseases and cancer, or alteration in the immune system able to induce the onset of neoplasms concomitant with the development of SLE, could be present in these female patients (6).

Apart from bilateral breast cancer, our patient also suffered from autoimmune thyroiditis. Several authors showed a high prevalence of ANA in patients with AT compared to healthy controls. SLE and AT probably share an underlying immunogenetic mechanism. Further evidence of the close association between SLE and AT is that the discontinuation of lupus therapy has been reported to be associated with relapse of thyrotoxicity (10). In addition to AT, it is not uncommon for SLE patients to present other autoimmune diseases and they must be carefully followed for the development of polyautoimmunity $(11,12)$.

In conclusion, the experience with our patient leads us to assert that SLE could be a "chaperone" of other disorders, and a complete evaluation, searching for other autoimmune diseases and hidden neoplasms, should be taken into consideration from case to case. 


\section{References}

1. Rider V, Abdou NI. Gender differences in autoimmunity: molecular basis for estrogen effects in systemic lupus erythematosus. Int Immunopharmacol. 2001;1: 1009-24.

2. Ganzetti G, Campanati A, Offidani A. Alopecia areata: a possible extraintestinal manifestation of Crohn's disease. J Crohns Colitis. 2012;6:962-3.

3. Nitzan O, Elias M, Saliba WR. Systemic lupus erythematosus and inflammatory bowel disease. Eur J Intern Med. 2006;17:313-8.

4. Campanati A, Giuliodori K, Ganzetti G, Liberati G, Offidani AM. A patient with psoriasis and vitiligo treated with etanercept. Am J Clin Dermatol. 2010;11 Suppl 1:46-8.

5. Cao L, Tong H, Xu G, Liu P, Meng H, Wang J, et al. Systemic lupus erythematous and malignancy risk: a meta-analysis. PLoS One. 2015;17;10.

6. Bernatsky S, Ramsey-Goldman R, Foulkes WD, Gordon C, Clarke AE. Breast, ovarian, and endometrial malignancies in systemic lupus erythematosus: a meta-analysis. Br J Cancer. 2011;26;104:1478-81.

7. Beral V, Bull D, Green J, Reeves G, Million Women Study Collaborators. Ovarian cancer and hormone replacement therapy in the Million Women Study. Lancet. 2007;369:1703-10.
8. Tessier Cloutier B, Clarke AE, Ramsey-Goldman R, Wang Y, Foulkes W, Gordon C, Hansen JE, et al. Breast cancer in systemic lupus erythematosus. Oncology. 2013;85:117-21.

9. Bernatsky S, Kale M, Ramsey-Goldman R, Gordon C, Clarke AE. Systemic lupus and malignancies. Curr Opin Rheumatol. 2012;24:177-81.

10. Dhir R, Ahluwalia Ai, Sridhar J, Mani H, Pruthi HS, Shah KM. Autoimmune thyroiditis perdating $[\mathrm{sic}]$ the presentation of systemic lupus erythematosus: two cases and review of literature. Indian J Dermatol Venereol Leprol. 2002;68:292-4.

11. Rojas-Villarraga A, Toro CE, Espinosa G, Rodríguez-Velosa Y, Duarte-Rey C, Mantilla RD, et al. Factors influencing polyautoimmunity in systemic lupus erythematosus. Autoimmun Rev. 2010;9:229-32.

12. Tektonidou MG, Anapliotou M, Vlachoyiannopoulos P, Moutsopoulos HM. Presence of systemic autoimmune disorders in patients with autoimmune thyroid diseases. Ann Rheum Dis. 2004;63:1159-61. 